\title{
Association of erythrocyte n-3 fatty acids with DXA-body fat and cardio-metabolic indices in middle-aged and elderly adults: a prospective study
}

\author{
Yuming Chen, Fang-fang Zeng, Jie-sheng Lin, Gengdong Chen, Ding Ding and Yi-hong Li \\ Sun Yat-sen University, Guangzhou, China
}

\begin{abstract}
Introduction: Many clinical trials showed favorable effects of high-doses supplemental n-3 polyunsaturated fatty acids (PUFA) on cardio-metabolic risk factors. However, limited studies examined the prospective associations of circulating n-3 PUFA with body fat and its distribution, metabolic syndrome (MS), carotid atherosclerosis, and nonalcoholic fatty liver disease (NAFLD) in subjects with habitual diets containing low levels of n-3 PUFA.
\end{abstract}

Materials and Methods: This community-based prospective study enrolled 4048 participants (40-75 years) at baseline (2008-2010, 2013) from Guangzhou, China. They were followed-up approximately once every 3 years. Fatty acids in erythrocyte membranes were measured at baseline. We determined metabolic syndrome factors, body fat by DXA scanning, carotid intima-media thickness (IMT) and NAFLD by ultrasound at the visits. General information, anthropometric indices, habitual dietary intake and other covariates were assessed at each visit.

Results: Among the total 4048 subjects, 3075 and 2671 subjects had erythrocyte n-3 PUFA data and completed the first and second follow-ups. Generally, erythrocyte n-3 PUFA were favorably associated with body fat (particularly at abdomen) and its changes, and with the presence and incidence of MS, type 2 diabetes, carotid IMT thickening. The participants with the highest (vs lowest) quartile of $n-3$ PUFA were associated with $-5.81 \%$ fat mass $(\mathrm{p}<0.001)$ and $-2.11 \%$ of fat mass change at the abdomen (Android) area. The adjusted hazards ratios $(95 \% \mathrm{CI})$ for the highest (vs. lowest) group were $0.74(0.61,0.89)$ (total n-3 PUFA), 0.71 (0.59, 0.86) (docosahexaenoic acid, DHA), 0.78 (0.65, 0.95) (docosapentaenoic acid, DPA), $1.96(1.60,2.40)$ (gamma-linolenic acid, GLA) for MS; 0.70 $(0.55,0.90)$ (total n-3 PUFA), 0.67(0.52,0.87) (DHA) and 0.73(0.57,0.93) (DPA) for bifurcation IMT thickening, 0.57(0.38, 0.86) (eicosapentaenoic acid, EPA) and $0.63(0.41,0.95)$ (DPA) for type 2 diabetes, and 1.18 (1.09, 1.33) (DHA) for alleviated NAFLD. Both higher levels of total and individual marine n-3 PUFAs (DHA, EPA and DPA) were associated with lower blood pressure at baseline and lower changes in diastolic and systolic blood pressure over the follow-up period. Plant n-3 PUFA ( $\alpha$-linolenic acid, ALA) largely had less significant association with the above-mentioned indices as compared with marine $n-3$ PUFAs.

Discussion: Higher proportions of erythrocyte n-3 PUFA (particularly marine sources) was associated with lower body fat, blood pressure and their changes, and lower risks of MS, type 2 diabetes and bifurcation IMT thickening, but higher chance of alleviated NAFLD in middle-aged and older adults.

\section{Conflict of Interest}

There is no conflict of interest 